\title{
Órteses de membros superiores para pessoas com lesão medular a nível cervical: revisão integrativa
}

\author{
Upper limb orthoses for people with cervical spinal cord \\ injury: an integrative review
}

\section{Ortesis de miembros superiores para personas con lesión de la médula espinal cervical: una revisión integradora}

\author{
Mariana Francisco Ramos ${ }^{1}$, Haidar Tafner Curi ${ }^{2}$, Cindy Passeti da \\ Costa Vida ${ }^{3}$, Francis Meire Favero ${ }^{4}$, Eliana Chaves Ferretti ${ }^{5}$
}

\begin{abstract}
1.Terapeuta Ocupacional, Discente do curso de pós-graduação em Neurologia Clínica, Universidade Federal de São Paulo - Campus São Paulo/SP, Brasil. ORCID: https://orcid.org/0000-0003-0771-7576

2.Terapeuta Ocupacional, Discente do curso de pós-graduação Interdisciplinar em Ciências da Saúde (mestrado), Universidade Federal de São Paulo - Campus Baixada Santista/Santos-SP, Brasil. ORCID: http://orcid.org/0000-0003-2660-2870

3.Terapeuta Ocupacional, Mestre em Ciências da Saúde, Universidade Federal de São Paulo - Campus Baixada Santista/Santos-SP, Brasil. ORCID: https://orcid.org/0000-0002-8522-4913

4.Professora Afiliada do Departamento de Neurologia/Neurocirurgia. Pesquisador do laboratório de Tecnologia da Inovação em Saúde. Universidade Federal de São Paulo - Campus São Paulo/ SP, Brasil. ORCID: https://orcid.org/0000-0001-8063-8167

5.Professor adjunto, Universidade Federal de São Paulo, Departamento de Ciências do Movimento Humano - Campus Baixada Santista/Santos-SP, Brasil. ORCID:

http://orcid.org/0000-0002-7605-828X
\end{abstract}

\section{Resumo}

Introdução. A lesão medular (LM) a nível cervical provoca diminuição ou perda da funcionalidade das extremidades e tronco, gerando alto grau de dependência nas atividades de vida diária (AVD's). O uso de órteses de membros superiores (MMSS) pode auxiliar na função do membro e promover o aumento da participação e funcionalidade da pessoa com LM. Objetivo. Identificar as características das órteses de MMSS indicadas para pessoas com LM a nível cervical. Método. Buscou-se nas fontes de indexação Pubmed, LILACS e Scielo estudos no idioma inglês e português, publicados entre os anos de 2000 a 2020. Os estudos foram avaliados e classificados de acordo com níveis de evidência científica. Resultados. Cinco estudos foram selecionados, destes quatro foram classificados como nível $\mathrm{V}$ e um como nível VI. Foram identificados os seguintes tipos de órteses de MMSS: órtese de punho lateral com tenodese por meio da extensão de punho; órtese dobradiça flexora acionada por punho; órtese acionada por punho impressa em 3D; Órtese de mão mioelétrica impressa em 3D; órtese híbrida semi-ativa de cotovelo associada ao uso de eletroestimulação funcional. As órteses apresentaram por finalidade promover a preensão de dedos e movimento de oposição do polegar e indicador e dedo médio por tenodese. Conclusão. Os tipos de órteses de MMSS demonstraram-se eficazes quanto à sustentação da preensão manual de objetos em indivíduos com LM cervical e promoveram melhora da funcionalidade nas AVD's.

Unitermos. Lesão medular; órtese; prática baseada em evidência; tetraplegia; tecnologia assistiva

\footnotetext{
Abstract

Introduction. The spinal cord injury (SCI) at the cervical level causes a decrease or loss of functionality of the extremities and trunk, generating a high degree of dependence on activities of daily living (ADLs). The use of upper limb orthoses (UL) can assist in limb function and promote increased participation and functionality for the person with SCI. Objective. To identify the characteristics of UL orthoses indicated for people with SCI at the cervical level. Method. Pubmed, LILACS and Scielo indexed sources were searched for studies in English and
} 
Portuguese, published between 2000 and 2020. The studies were evaluated and classified according to levels of scientific evidence. Results. Five studies were selected, of these four were classified as level V and one as level VI. The following types of upper limb orthoses were identified: lateral wrist orthosis with tenodesis through wrist extension; wrist-operated flexor hinge orthosis; 3D printed wrist-operated orthosis; 3D printed myoelectric hand orthosis; semi-active hybrid orthosis of the elbow associated with the use of functional electrostimulation. The purpose of the orthoses was to promote finger grasping and opposition movement of the thumb and index and middle fingers by tenodesis. Conclusion. The types of UL orthoses proved to be effective in sustaining handgrip of objects in individuals with cervical SCI and promoted an improvement in functionality in the ADLs.

Keywords. Spinal cord injury; orthosis; evidence-based practice; quadriplegia, assistive technology

\begin{abstract}
Resumen
Introducción. La lesión medular (LM) a nivel cervical provoca una disminución o pérdida de la funcionalidad de las extremidades y el tronco, generando un alto grado de dependencia de las actividades de la vida diaria (AVD). El uso de ortesis de miembros superiores (MMSS) puede ayudar en la función del miembro, y promover una mayor participación y funcionalidad de la persona con LM. Objetivo. Identificar las características de las ortesis de MMSS indicadas para personas con LM a nivel cervical. Método. Se realizaron búsquedas en las fuentes de indexación de Pubmed, LILACS y Scielo para estudios en inglés y portugués, publicados entre 2000 y 2020. Los estudios fueron evaluados y clasificados según niveles de evidencia científica. Resultados. Se seleccionaron cinco estudios, de estos cuatro se clasificaron como nivel $\mathrm{V}$ y uno como nivel VI. Se identificaron los siguientes tipos de ortesis de miembros superiores: ortesis de muñeca lateral con tenodesis mediante extensión de muñeca; órtesis de bisagra flexora accionada por muñeca; Órtesis accionada por muñeca impresa en 3D; Ortesis de mano mioeléctrica impresa en 3D; Ortesis de codo híbrida semiactiva asociada al uso de electroestimulación funcional. El propósito de las ortesis era promover el agarre de los dedos y el movimiento de oposición del pulgar y los dedos índice y medio mediante tenodesis. Conclusión. Se ha demostrado que los tipos de ortesis de MMSS son eficaces para sostener el agarre manual de objetos en personas con LME cervical y tienen una funcionalidad mejorada en las AVD.
\end{abstract}

Palabras clave: Lesión medular; ortesis; Práctica Basada en la Evidencia; cuadriplejia; tecnología de asistencia

Trabalho realizado para conclusão de curso de pós graduação em Neurologia Clínica da Universidade Federal de São Paulo, Campus São Paulo, São Paulo-SP, Brasil.

\title{
INTRODUÇÃO
}

A lesão medular (LM) é considerada como qualquer dano causado à medula espinhal. De acordo com a Organização Mundial de Saúde (OMS), é estimado por ano 40 a 80 casos por milhão de habitante ${ }^{1}$. No Brasil, a incidência varia de 16 a 26 casos por milhão/ano, sendo o 
segmento cervical o mais afetado, representando aproximadamente $37 \%$ dos casos ${ }^{2}$.

A LM a nível cervical, completa ou incompleta, pode provocar diminuição ou perda da funcionalidade das extremidades e tronco, gerando alto grau de dependência nas atividades de vida diária (AVD) ${ }^{3}$. Pessoas com LM, ainda que mediante necessidades singulares, podem necessitar de equipamentos auxiliares para o desempenho de diversas atividades ${ }^{4}$. Estes equipamentos fazem parte de um grande conjunto de tecnologias denominado tecnologia assistiva (TA) - compreendidos como itens, equipamentos ou sistemas projetados comercialmente ou sob medida, com a finalidade de aumentar, manter ou resgatar a capacidade funcional de indivíduos 5 .

Dentre as tecnologias assistivas, a órtese caracterizase por ser um dispositivo aplicado externamente em algum segmento do corpo com a finalidade de auxiliar na prevenção de deformidades, manutenção da amplitude articular e da força muscular e potencialização da função do membro ${ }^{5}$. A confecção de órteses exige uma gama de conhecimentos que envolvem desde a escolha e conhecimento do material até a avaliação da estrutura anatômica, funcionalidade e necessidades de cada indivíduo, a fim de definir o modelo ideal a ser prescrito, bem como os recursos a serem utilizados para a confecção e posicionamento adequado ${ }^{5}$. Desta forma, a prescrição e confecção de órtese deve ser realizada por profissionais habilitados e capacitados, 
garantindo a efetividade do dispositivo mediante a individualidade e necessidades de cada indivíduo.

Segundo o Conselho Federal de Fisioterapia e Terapia Ocupacional (COFFITO), o terapeuta ocupacional é apontado como apto a confeccionar, prescrever, orientar e realizar treinos com o uso de órteses ${ }^{6}$. No processo de reabilitação da pessoa com LM, o terapeuta ocupacional busca por meio do uso da órtese, maximizar a função dos membros superiores (MMSS) e prevenir deformidades, facilitando a participação e o aumento do desempenho em atividades significativas.

Estudos anteriores demonstram que o uso da órtese pode auxiliar na função dos MMSS e promover maior participação e funcionalidade nas $A V D$ ' $\mathrm{s}^{7-9}$. As órteses podem ser indicadas para evitar deformidades e favorecer manutenção ou aumento da amplitude articular ${ }^{8}$. Ainda, possuem potencial para promover funcionalidade manual, sem um custo elevado e necessidade cirúrgica ${ }^{9}$. Em casos de pessoas com tetraplegia, comumente é realizado a indicação de órteses de substituição, as quais podem promover maior preensão controlada pelo punho, possibilitando a preensão de objetos de tamanhos variados ${ }^{7}$.

Estudos de revisão da literatura são essenciais para contribuir na eficácia da prática clínica, permitindo ao profissional da saúde identificar e realizar uma determinada intervenção baseada em evidências ${ }^{10}$. A revisão integrativa, método da prática baseada em evidência (PBE), possibilita analisar e descrever estudos com diversas abordagens 
metodológicas, contribuindo à compreensão acerca do tema central do estudo, atualização das evidências presentes na literatura e fundamentação à prática clínica ${ }^{11}$.

Apesar da literatura conter estudos que apresentem os benefícios de órteses às pessoas com tetraplegia, estudos de revisão de literatura são escassos. Sendo assim, se faz necessário analisar as evidências sobre os modelos de órteses de MMSS e suas finalidades com ênfase nas pessoas com LM a nível cervical. Portanto, por meio do método da revisão integrativa, o presente estudo tem como objetivo identificar as características das órteses de MMSS indicadas às pessoas com LM à nível cervical.

\section{MÉTODO}

A revisão integrativa, método da prática baseada em evidência (PBE), permite analisar e sintetizar 0 conhecimento sobre um determinado tema, classificando-o de acordo com os níveis da evidência científica ${ }^{11}$. O processo de elaboração da revisão integrativa possui 6 etapas: 1) elaboração da questão norteadora; 2) busca ou amostragem na literatura; 3) coleta de dados; 4) análise crítica dos estudos incluídos; 5) discussão dos resultados e 6) apresentação da revisão integrativa ${ }^{11}$.

Sendo assim, teve por base a seguinte questão norteadora: quais as características das órteses de MMSS indicadas às pessoas com LM à nível cervical? Para tal, realizou-se a busca de estudos na base de dados MEDLINE 
via PubMed, LILACS e SCIELO na língua inglesa e portuguesa, publicados entre os anos de 2000 a 2020. Utilizou-se os descritores de acordo com o DeCS (Descritores em Ciências da Saúde) e termos, os quais são significativos ao tema: spinal cord injury (lesão medular), orthosis (órtese) e upper limb (membro superior), Tetraplegic (tetraplegia). Foram os utilizados os booleanos "AND" e "OR" combinados com os descritores e termos no idioma inglês e português da seguinte forma: "spinal cord injury OR tetraplegic" (lesão medular AND tetraplegia), "orthosis AND upper limb" (órteses AND membro superior).

Como critérios de inclusão, no processo de busca foram considerados: estudos que investigaram o uso da órtese de MMSS em pessoas com LM a nível cervical; estudos com grupo amostral com idade média entre 18 a 60 anos e artigos de periódicos publicados nos idiomas português e inglês. Como critérios de exclusão, não foram considerados: artigos editoriais; estudos de simulação; e revisões narrativas.

Os dados coletados a partir dos estudos selecionados foram sistematizados em formato de tabela. Foram coletados os seguintes dados: autor/ano/origem; design de estudo/nível de evidência; número da amostra/idade média; tipo de órtese; função da órtese; e desfecho principal. Todos os estudos foram avaliados criteriosamente por dois revisores, baseando-se nos níveis da evidência científica fornecidos por Cook et al. ${ }^{12}$. A classificação da evidência científica é determinada de acordo com o desenho de estudo utilizado, sendo organizada hierarquicamente em sete 
níveis. Os níveis de evidência são classificados como: I Revisão sistemática; II - Estudos randomizados; III Estudos de Coorte; IV - Estudos caso Controle; V - Série de Casos; VI - Relato de Caso; VII - Opinião de Especialistas. As evidências dos artigos incluídos na revisão foram discutidas com os demais artigos presentes na literatura científica referente à temática.

\section{RESULTADOS}

\section{Características amostral dos estudos incluídos}

No total, 647 estudos foram identificados. Após a aplicação dos critérios de inclusão e exclusão, 11 estudos foram selecionados para a leitura na íntegra, dos quais 6 foram desconsiderados para o estudo, sendo que um não foi publicado em periódico, três não testaram a órtese em indivíduos com LM, um apresentou relato de órteses em membros inferiores e um não apresentou o uso da órtese como objeto central do estudo. Desta forma, cinco estudos foram incluídos na presente revisão integrativa (Figura 1).

Quatro estudos foram classificados como nível V13,15-17 e somente um como nível $\mathrm{VI}^{14}$. $\mathrm{O}$ desenho de estudo série de casos foi o mais utilizado dentre as produções. Os estudos incluídos foram originados nos Estados Unidos $(n=1)$, Alemanha $(n=1)$, Nova Zelândia $(n=1)$ e Coréia $(n=2)$. Na maior parte dos estudos os grupos amostrais foram constituídos predominantemente por participantes do sexo masculino, exceto no estudo de Portnova $2018^{16}$. 
Figura 1. Fluxograma dos estudos selecionados.

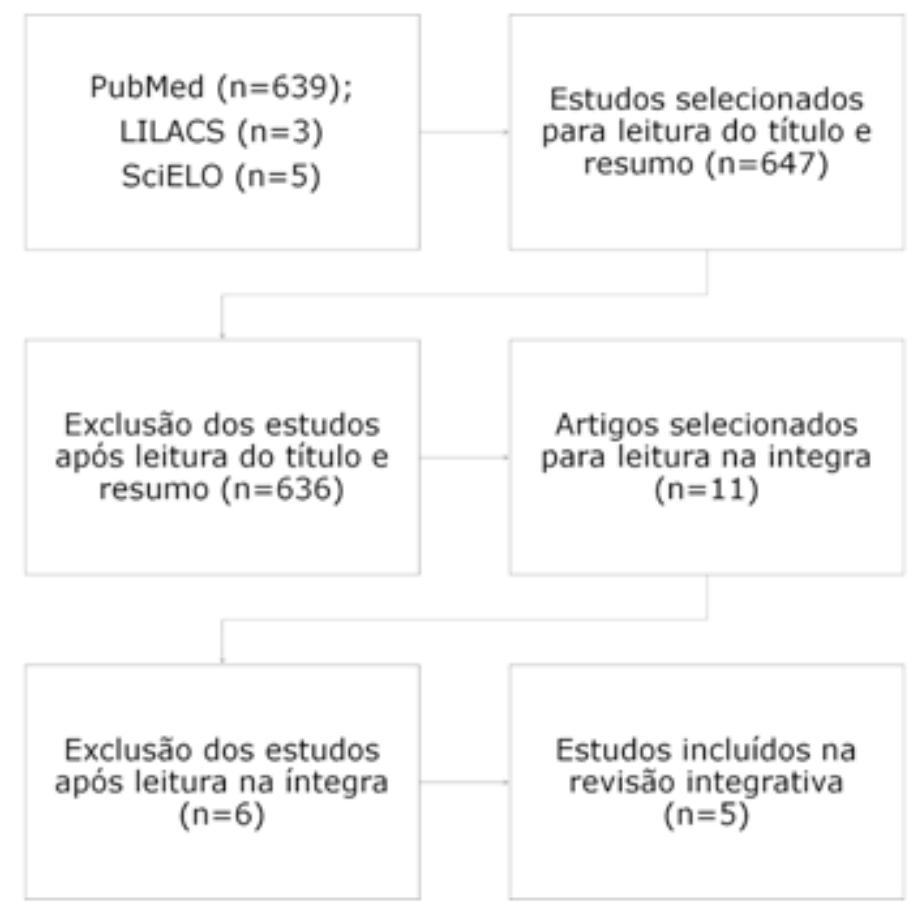

\section{Descrição dos estudos}

King $2009^{13}$ investigou o uso da órtese dinâmica de punho com a finalidade de promover pinça lateral. A órtese utiliza um cabo bowden, encontrado em bicicletas, que percorre o antebraço até a região palmar. Uma parte do cabo flexível é fixada a um anel situado na falange proximal do polegar, enquanto, a outra parte é fixada em uma espécie de almofada na região palmar. A extensão do punho causa tensão no cabo e, consequentemente, gera adução do polegar em direção a região lateral da falange proximal ou medial do dedo indicador, promovendo a pinça lateral. A órtese pode ser colocada e retirada de forma independente e o comprimento do cabo pode ser ajustado de acordo com a necessidade do usuário. Participaram do estudo seis pessoas com LM C5-C7, os quais utilizaram a órtese todos os 
dias por pelo menos trinta minutos durante seis semanas em tarefas funcionais. Foram realizadas mensurações com e sem órtese para verificar a força e capacidade funcional da preensão em pinça lateral. Como resultado, constatou-se aumento da força de preensão em pinça lateral e no número de tarefas realizadas com o uso da órtese. O estudo demonstrou que o uso da órtese pode beneficiar a função da mão em indivíduos com LM C5-C7 com extensão de punho grau 3 ou mais ${ }^{13}$. A órtese utilizada no estudo é protegida por patente ${ }^{18}$.

Rohm $2013^{14}$ investigou o uso de uma órtese dinâmica associada à eletroestimulação funcional (FES) e interface híbrida cérebro-computador para promover a função da mão, dedos e cotovelo de um indivíduo com LM nível C4. O treinamento com FES foi realizado durante dois meses por 30 a 40 minutos, duas ou três vezes por semana, associado a imagética motora e BCI - Brain Computer Interfaces, totalizando 43 semanas. Uma órtese dinâmica de cotovelo foi desenvolvida como um complemento do dispositivo de FES. Os principais componentes são um dispositivo de articulação do cotovelo com travamento automático/travável associado a um sistema de suporte de peso para suportar os movimentos de flexão e extensão de cotovelo. A órtese pode ser estendida por meio de um módulo rotatório de punho, um módulo para desvio ulnar-radial e um módulo estabilizador tenar de punho. O dispositivo permite que seja acomodado para diferentes necessidades funcionais e deve ser calibrado ao ser colocado. Após um ano, o usuário 
apresentou média de $70,5 \%$ de desempenho no BCI, avaliado através de funções como escrita e levar alimentos à boca. A órtese semiativa em modelo híbrido associado ao uso do FES e BCI permitiu função de cotovelo e dedos, favorecendo a realização das tarefas e seu desempenho permaneceu moderado, segundo descrito pelos avaliadores.

Kang $2013^{15}$ avaliou a órtese de dobradiça flexora acionada por punho, composta por três componentes articulados principais, antebraço, palmar e dedos. O dispositivo ativa a flexão dos dedos durante a extensão do punho, resultando em preensão de três pontos, neste caso com polegar imobilizado. A órtese permite que os dedos abram ou fechem conforme a movimentação do punho e conta com cinco níveis de regulagem de extensão do punho. $\mathrm{O}$ estudo contou com vinte e quatro participantes com LM em nível C6-C7. As medidas dos participantes foram tomadas antes do ensaio para o ajuste da órtese. $O$ teste foi realizado com e sem o uso da órtese na mão dominante e os movimentos foram mensurados por meio de eletromiografia. Os resultados demonstraram melhora da força de preensão de pinça com o uso da órtese e correlacionou-se com a força dos músculos extensores. No entanto, a eficiência da órtese diminuiu à medida que a contração voluntária máxima de extensores de punho aumentou. Verificou-se também que 0 desvio radial do punho, que pode ocorrer em decorrência da lesão, restringe a ação eficiente da órtese.

Portnova $2018^{16}$ desenvolveu uma órtese acionada por punho impressa em 3D para promover funções em 
atividades como escrita, pegar e virar um cartão e alimentação simulada. As medidas das estruturas dos MMSS dos participantes foram tomadas para determinar o tamanho apropriado do dispositivo impresso. A função foi avaliada com e sem órtese utilizando o teste Jebsen Taylor Hand Function (JTHF) e dinamômetro de preensão em pinça. Com uso da órtese, os três participantes do estudo apresentaram redução no tempo de execução da tarefa em transferir blocos e, dois melhoraram a força de pinça trípode. O estudo demonstrou que a órtese impressa em 3D é promissora devido ao baixo custo, leveza, estética (discreta), conforto, funcionalidade e aumento de força de preensão trípode.

Yoo $2019^{17}$ utilizou uma órtese de mão mioelétrica impressa em 3D associada a um motor linear acoplado à tala na região dorsal do antebraço. O dispositivo é ajustável por velcros na região dorsal para controlar extensão de punho, enquanto os velcros da região ventral estabilizam a articulação do punho. Anéis foram colocados nas falanges, do primeiro ao terceiro dedos e ligados ao antebraço por fios de nylon. Logo, quando o punho faz extensão, proporcionada pelo motor linear, fortalece a empunhadura de tenodese. Todas as partes da órtese foram impressas e ajustadas de acordo com o formato da mão do indivíduo. Os participantes foram avaliados com e sem o uso da órtese na mão dominante, utilizando-se o Toronto Rehabilitation Institute Hand Function Test (TRI-HFT) para verificar a capacidade de manipular objetos e a força de preensão. A funcionalidade em atividades diárias também foi avaliada, por meio da 
Medida de Independência Funcional (MIF) e Spinal Cord Independence Measure (SCIM). A órtese aprimorou a tenodese e apresentou melhorias na função da mão, com escores da TRI-HFT inicialmente de 29,40 e final de 45,00. Apesar do resultado promissor, esse tipo de órtese não foi projetada para movimentação individual dos dedos. Além disso, os participantes não apresentaram melhora significativa para preensão de objetos pequenos e/ ou planos. Por outro lado, observou-se melhora para atividade de alimentação na MIF e na SCIM. A órtese foi considerada leve e de baixo custo.

A Tabela 1 apresenta a síntese dos estudos incluídos na presente revisão integrativa.

\section{DISCUSSÃO}

A presente revisão integrativa forneceu evidências sobre as características das órteses de MMSS indicadas às pessoas com LM à nível cervical. A maioria das produções utilizaram desenho de estudo de série de $\operatorname{casos}^{13,15-17}$, comumente utilizado para investigar a efetividade de uma intervenção em um pequeno grupo de pessoas ${ }^{19}$. Apesar das importantes contribuições, o desenho série de casos é configurado com um nível de evidência inferior em relação às evidências provindas de outros desenhos de estudo, por exemplo, estudo de caso-controle ${ }^{12}$. Desta forma, é essencial que futuras investigações sejam desenvolvidas a partir de outras abordagens metodológicas, a fim de produzir maiores níveis de evidências sobre o tema. 
Tabela 1. Síntese dos estudos incluídos na revisão.

\begin{tabular}{cccccc}
\hline $\begin{array}{c}\text { Autor/ano } \\
\text { /origem }\end{array}$ & $\begin{array}{c}\text { Nível de } \\
\text { evidência }\end{array}$ & $\begin{array}{c}\text { Amostra/ } \\
\text { idade } \\
\text { média/ } \\
\text { diagnóstico }\end{array}$ & $\begin{array}{c}\text { Tipos de } \\
\text { órteses }\end{array}$ & $\begin{array}{c}\text { Finalidade } \\
\text { das órteses }\end{array}$ & $\begin{array}{c}\text { Desfecho } \\
\text { principal do } \\
\text { estudo }\end{array}$ \\
\end{tabular}

\begin{tabular}{|c|c|c|c|c|c|}
\hline $\begin{array}{l}\text { King } 2009^{13} \\
\text { Nova } \\
\text { Zelândia }\end{array}$ & $\mathrm{V}$ & $\begin{array}{c}\mathrm{n}=6 \\
\mathrm{ND} \\
\text { idade média } \\
\mathrm{C} 5-\mathrm{C} 7\end{array}$ & $\begin{array}{c}\text { Órtese } \\
\text { dinâmica de } \\
\text { punho }\end{array}$ & $\begin{array}{l}\text { Promover } \\
\text { preensão } \\
\text { lateral }\end{array}$ & $\begin{array}{l}\text { Maior força de } \\
\text { preensão lateral } \\
\text { e aumento no } \\
\text { número de } \\
\text { AVDs }\end{array}$ \\
\hline $\begin{array}{l}\text { Rohm } \\
2013^{14} \\
\text { Alemanha }\end{array}$ & VI & $\begin{array}{c}n=1 \\
41 \text { anos } \\
\text { C4 }\end{array}$ & $\begin{array}{c}\text { Órtese } \\
\text { dinâmica } \\
\text { associada a } \\
\text { FES e } \\
\text { interface } \\
\text { híbrida } \\
\text { cérebro- } \\
\text { computador }\end{array}$ & $\begin{array}{c}\text { Promover } \\
\text { função de } \\
\text { mão, dedos e } \\
\text { cotovelo }\end{array}$ & $\begin{array}{l}\text { Aumento da } \\
\text { funcionalidade } \\
\text { da escrita e } \\
\text { para levar } \\
\text { alimentos à } \\
\text { boca }\end{array}$ \\
\hline
\end{tabular}

\begin{tabular}{|c|c|c|c|c|}
\hline $\begin{array}{l}\text { Kang201315 } \\
\text { Coréia }\end{array}$ & $\mathrm{V}$ & $\begin{array}{c}\mathrm{n}=24 \\
37,1 \pm 12,8 \\
\text { C6-C7 }\end{array}$ & $\begin{array}{c}\text { Órtese } \\
\text { flexora } \\
\text { acionada por } \\
\text { punho }\end{array}$ & $\begin{array}{c}\text { Promover } \\
\text { oposição do } \\
\text { polegar e } \\
\text { indicador e } \\
\text { dedo médio } \\
\text { por tenodese }\end{array}$ \\
\hline
\end{tabular}
Aumento da
força de pinça, correlacionada positivamente com a força dos músculos extensores do punho durante a tenodese

\begin{tabular}{|c|c|c|c|}
\hline $\begin{array}{l}\text { Portnova } \\
2018^{16} \\
\text { EUA }\end{array}$ & $\mathrm{V}$ & $\begin{array}{c}n=3 \\
51,3 \pm 10,26 \\
\text { C4-C6 e C5- } \\
\text { C6 }\end{array}$ & $\begin{array}{l}\text { Órtese de } \\
\text { punho por } \\
\text { meio da } \\
\text { impressão } \\
\text { 3D }\end{array}$ \\
\hline
\end{tabular}

3D

Melhora na capacidade de desempenho em AVD's como

Promover Melhora na
preensão de todos os dedos e preensão trípode. escrita e alimentação. Aumento da força trípode

\begin{tabular}{|c|c|c|c|c|c|}
\hline $\begin{array}{l}\text { Yoo } 201917 \\
\text { Coréia do } \\
\text { Sul }\end{array}$ & $\mathrm{V}$ & $\begin{array}{c}n=10 \\
49,5 \pm 10,6 \\
C 4-C 7\end{array}$ & $\begin{array}{l}\text { Órtese } \\
\text { mioelétrica } \\
\text { por meio da } \\
\text { impressão } \\
\text { 3D }\end{array}$ & $\begin{array}{l}\text { Promover } \\
\text { preensão } \\
\text { através da } \\
\text { tenodese }\end{array}$ & $\begin{array}{c}\text { Melhora na } \\
\text { função durante } \\
\text { a atividade } \\
\text { comer; } \\
\text { Aumento da } \\
\text { preensão de } \\
\text { objetos gerada } \\
\text { por } \\
\text { tenodese; sem } \\
\text { melhoras } \\
\text { significativas } \\
\text { para objetos } \\
\text { pequenos }\end{array}$ \\
\hline
\end{tabular}

AVD 's = Atividades de Vida Diária; FES = Estimulação Elétrica Funcional; ND = Não descrito. 
Todos os estudos apresentaram como objetivo principal promover maior desempenho da função de preensão manual por meio do uso das órteses de MMSS. Em detalhes, as produções investigaram o uso de órteses dinâmicas, caracterizadas por auxiliar ou substituir a ausência da força muscular, por meio da aplicação de forças constantes em determinados segmentos corporais. As órteses dinâmicas são comumente indicadas às pessoas com LM, uma vez que, devido ao trauma, apresentam diminuição ou ausência da força muscular dos MMSS ${ }^{7}$. Por meio do mecanismo de tenodese, movimento de extensão do punho que produz flexão dos dedos da mão, a órtese dinâmica potencializa a aplicação de forças constantes e, consequentemente, favorece maior desempenho na preensão de objetos ${ }^{5}$. Em outras palavras, quando o movimento de extensão do punho é preservado, a órtese aproxima os dedos, gerando ação de preensão do objeto, e assim, possibilita a realização de atividades como higiene, escrita e alimentação ${ }^{20}$.

O uso da órtese dinâmica demonstrou aumento da força nas preensões de pinça, trípode e chave gerada por meio do mecanismo de tenodese, porém sem melhoras significativa para objetos pequenos; melhora funcional nas AVD's, principalmente na alimentação, para levar alimentos à boca, e funcionalidade durante a escrita. Ainda, compreende-se que os avanços tecnológicos em impressões 3D permitiram meios para favorecer maior independência e autonomia, utilizando o mecanismo de tenodese como base para o desenvolvimento dispositivo. Recentemente, as 
investigações sobre o uso de tecnologia de impressão em 3D para confecção de órteses de MMSS às pessoas com LM tem aumentado significativamente em busca de maior agilidade de produção e maior precisão na confecção de dispositivos mais avançados. A órtese de impressão 3D pode ter valor de produção reduzido, a depender do tipo de material escolhido, podem ser também personalizadas para cada indivíduo, permite definir a estética e bom ajuste ${ }^{21}$. Órteses de impressão 3D foram descritas em dois dos estudos ${ }^{16,17}$, demonstrando ganho de funcionalidade, percepção de maior conforto e aceitação do uso do dispositivo pelos usuários. Por outro lado, sabe-se que as observações sobre o uso de órtese desses estudos não se caracterizaram como longitudinais, isto é, os resultados encontrados em um único período podem ser diferentes quando há acompanhamento a longo prazo. Sendo assim, é essencial que futuras investigações realizem estudos longitudinais, para avaliar as condições das órteses prescritas, fornecer treinamento e suporte aos usuários com intuito de diminuir a possibilidade de abandono do dispositivo assistivo ${ }^{22}$.

Apenas Rohm $2013^{14}$ utilizou em seu estudo uma órtese híbrida, isto é, uma órtese semiativa de cotovelo associada à eletroestimulação. A eletroestimulação representa um dos esforços mais influentes na área de reabilitação em tetraplegia com resultados em funcionalidade de preensão e restauração de capacidades em AVD's ${ }^{7}$. Em outro estudo, dois participantes com lesões em níveis C3 e C4 foram capazes de melhorar a função de alimentação com uso de 
órtese híbrida ${ }^{23}$, assim como apresentado por Rohm 2013 ${ }^{14}$, com nível de LM em C4, em que o indivíduo foi capaz de levar alimentos à boca e assinar documento com uso da órtese.

No Brasil é comum o uso de material termoplástico de baixa temperatura para a confecção de órteses ${ }^{24}$. No caso das órteses dinâmicas notamos que em sua maioria não é comum o envolvimento de toda a tecnologia presente nos artigos apresentados neste estudo. Tendo em vista os benefícios desse tipo de órtese, este estudo se faz pertinente como embasamento e incentivo para o desenvolvimento de órteses dinâmicas na reabilitação de pessoas com LM no Brasil. Acredita-se que os recursos oferecidos pela impressão 3D sejam promissores e possivelmente acessíveis à população. Para tanto, é de suma importância o desenvolvimento de novos estudos que possam tanto comprovar a eficácia como possibilitar o desenvolvimento desses recursos em território nacional.

Apesar dos promissores e relevantes resultados apresentados, algumas produções apontam a necessidade de aprimorar a funcionalidade das órteses, uma vez que se depararam com limitações de posicionamento e movimentos, como o aparecimento de desvio radial do punho, restringindo a eficiência da ação da órtese ${ }^{15}$, e limitação para preensão de objetos planos e/ou se pequenos $^{17}$. Além disso, seria necessário o desenvolvimento de estudos com um maior número de participantes com o objetivo de oferecer dispositivos acessíveis que atendam as 
demandas de pessoas com LM em nível cervical na execução das suas tarefas diárias.

\section{CONCLUSÃO}

As órteses dinâmicas para MMSS por meio do uso de tenodese demonstrou ser a mais utilizada e eficaz para indivíduos com tetraplegia devido a LM em nível cervical. Sugere-se que futuras investigações sejam desenvolvidas com um maior número de participantes com LM e com desenhos de estudos que favoreçam maior evidência científica. Correlacionar o uso das órteses dinâmicas de MMSS com a funcionalidade nas AVD's, especificando cada atividade, além da mensuração da força de preensão manual poderá fortalecer as evidências sobre a tema.

\section{REFERÊNCIAS}

1. World Health Organization (WHO). Spinal cord injury. Switzerland. (endereço na Internet). Geneva; 2013. (Acessado: 2020). Disponível em: who.int/news-room/fact-sheets/detail/spinal-cord-injury

2. Botelho RV, Gianini ALD, Junior RB, Arantes Júnior AA. Epidemiology of traumatic spinal injuries in Brazil: systematic review. Arq Bras Neurocir 2014;33:100-6. https://doi.org/10.1055/ s-0038-1626255

3.Brasil. Ministério da Saúde. Secretaria de Atenção à Saúde. Departamento de Ações Programáticas Estratégicas. Diretrizes de Atenção à Pessoa com Lesão Medular/Ministério da Saúde, Secretaria de Atenção à Saúde, Departamento de Ações Programáticas Estratégicas e Departamento de Atenção Especializada. (endereço na Internet0. Brasília: Ministério da Saúde; 2013. (Acessado em 2019). Disponível

em: https://bvsms.saude.gov.br/bvs/publicacoes/diretrizes atencao pess oa lesao medular.pdf

4.Arazpour M, Bani MA, Mousavi ME, Bahramizadeh M, Mardani MA. Orthoses for Spinal Cord Injury Patients. In: Intech Open. Recovery Motor Function Following Spinal Cord Injury 2016,259-276.

http://dx.doi.org/10.5772/64092 
5.Brasil. Ministério da Saúde. Secretaria de Atenção Especializada à Saúde. Guia para Prescrição, Concessão, Adaptação e Manutenção de Órteses, Próteses e Meios Auxiliares de Locomoção / Ministério da Saúde, Secretaria de Atenção Especializada à Saúde, Departamento de Atenção Especializada e Temática. (endereço na Internet). Brasília: Ministério da Saúde; 2019. (acessado em 2020 Jan 25). Disponível em: http://bvsms.saude.gov.br/bvs/publicacoes/guia manutencao ortese s proteses auxiliares locomocao.pdf

6. Conselho Federal de Fisioterapia e Terapia Ocupacional (COFFITO). Resolução No 458, de 20 de Novembro de 2015 - Dispõe sobre o uso da Tecnologia Assistiva pelo terapeuta ocupacional e dá outras providências. (Acessado em: 2019 Ago 25). Disponível em: https://www.coffito.gov.br/nsite/?p=3221

7.Tubbs J, Libra D. Upper limb orthoses for persons with spinal cord injuries and brachial plexus injuries. In: Atlas of Orthoses and Assistive Devices, 5th ed. Amsterdã: Elsevier; 2019; pp157-69.e4. https://www.sciencedirect.com/science/article/pii/B97803234832300 $\underline{00147}$

8.Gradim LCC, Paiva G. Modelos de órteses para membros superiores: uma revisão da literatura. Cad Bras Terap Ocupac 2018;26:479-88. https://doi.org/10.4322/2526-8910.ctoAR1174

9. Petroff N, Reisinger KD, Mason PAC. Fuzzy-control of a hand orthosis for restoring tip pinch, lateral pinch, and cylindrical prehensions to patients with elbow flexion intact. IEEE Trans Neural Syst Rehabil Eng 2001;9:225-31. https://doi.org/10.1109/7333.928582

10.Sampaio RF, Mancini MC. Estudos de revisão sistemática: um guia para síntese criteriosa da evidência científica. Rev Bras Fisioter 2007;11:83-9. https://doi.org/10.1590/s1413-35552007000100013

11.Souza MT, Silva MD, Carvalho R. Revisão integrativa: o que é e como fazer. Einstein 2010;8:102-6. https://doi.org/10.1590/s1679$45082010 \mathrm{rw} 1134$

12.Cook DJ, Guyatt GH, Laupacis A, Sackett DL, Goldberg DL. Clinical recommendations using levels of evidence for antithrombotic agents. Chest $1995 ; 108: 227-30$.

https://doi.org/10.1378/chest.108.4 supplement.227s

13.King MJ, Verkaaik JK, Angela NA, Collins F. A wrist extension operated lateral key grip orthosis for people with tetraplegia. Technol Disabil 2009;21:19-23. https://doi.org/10.3233/TAD-2009-0267 14.Rohm M, Schneiders M, Müller C, Kreilinger A, Kaiser V. Hybrid brain computer interfaces and hybrid neuroprostheses for restoration of upper limb functions in individuals with high spinal cord injury. Artif Intell Med 2013;59:133-42.

https://doi.org/10.1016/j.artmed.2013.07.004

15.Kang YS, Park YG, Lee BS, Park HS. Biomechanical evaluation of wrist-driven flexor hinge orthosis in persons with spinal cord injury. J Rehabil Res Dev 2013;50:1129-38.

https://doi.org/10.1682/JRRD.2012.10.0189

16.Portnova AA, Mukherjee G, Peters KM, Yamane A, Steele $\mathrm{KM}$. Design of a 3D-printed, open-source wrist-driven orthosis for 
individuals with spinal cord injury. PLoS One 2018;13:1-18. https://doi.org/10.1371/journal.pone.0193106

17.Yoo HJ, Lee S, Kim J, Park C, Lee B. Development of 3D-printed myoelectric hand orthosis for patients with spinal cord injury. J NeuroEngineer Rehabil 2019;16:162.

https://doi.org/10.1186/s12984-019-0633-6

18.King MJ, Le-Ngoc L, Verkaaik j. An Orthosis - PCT International patent application PCT/NZ2006/000160 and NZ patent application 540912. World Intellectual Property Organization patent, WIPO WO2006137748A1. 2006. (acessado em 2020). Disponível em: https://patents.google.com/patent/WO2006137748A1/fr

19.Parente RCM, Oliveira MAP, Celeste RK. Relato de série de casos na era da medicina baseada em evidência. Bras J Vídeo-Sur 2010; 42:6770. https://doi.org/10.1590/0100-69912015005001

20.Edelstein J, Bruckner J. Órteses: abordagem clínica. Rio de Janeiro: Guanabara Koogan, 2006.

21.Chae DS, Kim D-H, Kang KY, Kim DY, Park SJ, Kim JH. O efeito funcional da órtese individualizada de impressão 3D para pacientes com lesões de nervos periféricos. Med 2020;99:e19791. https://doi.org/10.1097/MD.0000000000019791

22. Federici S, Mele ML, Romeo AS, Didimo W, Liotta G, Borsci S, et al. A Model of Web-Based Follow-Up to Reduce Assistive Technology Abandonment. In: Kurosu M (eds). Human-Computer Interaction. Applications and Services. HCI 2014. Lecture Notes in Computer Science, vol 8512. Amsterdã: Springer. https://doi.org/10.1007/9783-319-07227-2_64

23.Benjuya N, Kenney SB. Hybrid Arm Orthosis. JPO 1990;2:155-63. https://insights.ovid.com/prostheticsorthotics/jport/1990/01/000/hybrid-arm-orthosis/12/00008526

24.Agnelli LB, Toyoda CY. Estudo de materiais para confecção de órteses e sua utilização prática por terapeutas ocupacionais no Brasil. Cad Terap Ocupac UFSCar 2003;11:83-94. http://www.cadernosdeterapiaocupacional.ufscar.br/index.php/cader nos/article/view/194 